ARTICLE

https://doi.org/10.1038/s41467-019-09290-y

\title{
Cascade anchoring strategy for general mass production of high-loading single-atomic metal-nitrogen catalysts
}

\author{
Lu Zhao ${ }^{1,2}$, Yun Zhang ${ }^{1,3}$, Lin-Bo Huang 1,2, Xiao-Zhi Liu'2,4, Qing-Hua Zhang ${ }^{4}$, Chao He ${ }^{1,2}$, Ze-Yuan Wu ${ }^{1,2}$, \\ Lin-Juan Zhang ${ }^{5}$, Jinpeng Wu (10 ${ }^{6}$, Wanli Yang (i) ${ }^{6}$, Lin Gu (i) ${ }^{4}$, Jin-Song Hü ${ }^{1,2} \&$ Li-Jun Wan ${ }^{1,2}$
}

Although single-atomically dispersed metal- $\mathrm{N}_{\mathrm{x}}$ on carbon support (M-NC) has great potential in heterogeneous catalysis, the scalable synthesis of such single-atom catalysts (SACs) with high-loading metal- $\mathrm{N}_{\mathrm{x}}$ is greatly challenging since the loading and single-atomic dispersion have to be balanced at high temperature for forming metal- $\mathrm{N}_{\mathrm{x}}$. Herein, we develop a general cascade anchoring strategy for the mass production of a series of M-NC SACs with a metal loading up to $12.1 \mathrm{wt} \%$. Systematic investigation reveals that the chelation of metal ions, physical isolation of chelate complex upon high loading, and the binding with $\mathrm{N}$-species at elevated temperature are essential to achieving high-loading M-NC SACs. As a demonstration, high-loading Fe-NC SAC shows superior electrocatalytic performance for $\mathrm{O}_{2}$ reduction and $\mathrm{Ni}-\mathrm{NC}$ SAC exhibits high electrocatalytic activity for $\mathrm{CO}_{2}$ reduction. The strategy paves a universal way to produce stable M-NC SAC with high-density metal- $\mathrm{N}_{\mathrm{x}}$ sites for diverse high-performance applications.

\footnotetext{
${ }^{1}$ Beijing National Laboratory for Molecular Sciences (BNLMS), CAS Key Laboratory of Molecular Nanostructure and Nanotechnology, Institute of Chemistry, Chinese Academy of Sciences, Beijing 100190, China. ${ }^{2}$ University of Chinese Academy of Sciences, Beijing 100049, China. ${ }^{3}$ College of Chemistry and Materials Science, Sichuan Normal University, Chengdu 610068, China. ${ }^{4}$ Beijing National Research Center for Condensed Matter Physics, Collaborative Innovation Center of Quantum Matter, Institute of Physics, Chinese Academy of Sciences, Beijing 100190, China. ${ }^{5}$ Shanghai Synchrotron Radiation Facility, Shanghai Institute of Applied Physics, Chinese Academy of Sciences, Shanghai 201800, China. ${ }^{6}$ Advanced Light Source, Lawrence Berkeley National Laboratory, Berkeley, California 94720, USA. These authors contributed equally: Lu Zhao, Yun Zhang. Correspondence and requests for materials should be addressed to J.-S.H. (email: hujs@iccas.ac.cn)
} 
S ingle-atom catalyst (SAC) has recently emerged as a risingstar in catalysis since it combines the merits of both heterogeneous and homogeneous catalysts while bridges the gap between them with unique features. Comparing with heterogeneous catalysts, SAC maximizes the atom utilization and has homogenous active sites with tunable electronic environments for highly catalytic activity or/and selectivity, while simultaneously holds improved stability and excellent recyclability in contrast to homogeneous catalysts ${ }^{1-10}$. In the past a couple of years, dozens of SACs have been therefore developed for thermocatalytic reaction (such as CO oxidation ${ }^{11,12}$, water-gas shift reaction ${ }^{12,13}$, and methane conversion ${ }^{14}$ ), photocatalytic reaction (photocatalytic $\mathrm{H}_{2}$ evolution ${ }^{15}$ and $\mathrm{CO}_{2}$ reduction ${ }^{16}$ ), electrocatalytic reaction $\left(\mathrm{H}_{2}\right.$ evolution ${ }^{17-19}, \mathrm{O}_{2}$ reduction ${ }^{20-24}, \mathrm{CO}_{2}$ reduction ${ }^{25}$, and $\mathrm{N}_{2}$ reduction ${ }^{26}$ ), as well as organic electrosynthesis ${ }^{27}$. Controllable preparation of SAC, however, still remains challenging in view of the strong tendency of migration and aggregation of active atoms during either the fabrication or the subsequent application processes. To this end, supporting monodispersed atoms on appropriate support represents the most feasible and effective way to achieve SAC. Until now, several strategies such as using confinement effect, coordination effect, or chemical bonding have been reported to synthesize isolated metal sites over supports by (1) limiting the loading amount of active component; (2) boosting the interactions between metal atom and support; or (3) employing defect or void on support ${ }^{28-32}$.

Among SACs, atomic metal- $\mathrm{N}_{\mathrm{x}}\left(\mathrm{M}-\mathrm{N}_{\mathrm{x}}\right)$ moieties anchored on carbon support (M-NC) have attracted particular interests especially in electrocatalysis, since nitrogen can not only effectively anchor and stabilize single-metal atom on carbon but also modulate the electronic structures of metal or carbon atom to optimize the adsorption/desorption of intermediates for enhancing catalytic performance ${ }^{33-37}$. Moreover, carbon supports are readily available for commercial use and highly electrically conductive for accelerating electron transfer during reactions. Such single-atomic M-NC SACs have demonstrated extraordinary promise in catalytic oxidation of benzene to phenol ${ }^{38}$, chemoselective hydrogenation of nitroarenes to produce azo compounds ${ }^{39}$, semihydrogenation of 1 -hexyne ${ }^{40}$, etc. Huang and
Duan et al. $^{41}$ recently reported a two-step approach to the synthesis of well-defined atomic $\mathrm{MN}_{4} \mathrm{C}_{4}(\mathrm{M}=\mathrm{Fe}, \mathrm{Co}, \mathrm{Ni})$ moieties embedded in graphene with a metal loading of $\sim 0.05$ at $\%$ as efficient electrocatalysts for oxygen evolution reaction.

The catalytic activity and turnover efficiency of a catalyst and thus the power/energy density of catalyst-based devices closely depend on the number of catalytic sites, besides its intrinsic activity. One of big challenges for SAC is the low concentration of single-atomic sites, since the loading and the aggregation of atoms have to be balanced. Especially at elevated temperature, metal atoms are getting easier to migrate and aggregate, causing more challenging to achieve M-NC SACs with high metal loading since the formation of $\mathrm{M}-\mathrm{N}$ bonding usually needs high temperature (such as over $\left.700^{\circ} \mathrm{C}\right)^{42-45}$. Although the progress has been made on the synthesis of M-NC SACs, few reports can achieve the metal loading over $4 \mathrm{wt} \%$. Another challenge is the mass production of M-NC SACs, which is essential to their practical applications. Most strategies for the synthesis of SAC need delicate control of the defects in supports and synthetic procedures to stabilize single atoms, whereas the mass production requires the commercially available low cost supports and scalable, manageable processing. It is therefore highly desirable to develop a method compatible with large-scale production for synthesizing M-NC SACs with high metal loadings.

Herein, we report a general approach to synthesize a wide range of $\mathrm{M}-\mathrm{NC}$ SACs $(\mathrm{M}=\mathrm{Mn}, \mathrm{Fe}, \mathrm{Co}, \mathrm{Ni}, \mathrm{Cu}, \mathrm{Mo}, \mathrm{Pt}$, etc.) with metal loadings up to $12.1 \mathrm{wt} \%$ via a cascade anchoring strategy. As shown in Fig. 1, the metal ions are first chelated by chelating agent such as glucose here, then anchored onto oxygen-speciesrich porous carbon support with high surface area (Step 1). The chelating agent can effectively sequester metal ions (primary protection) and bind to O-rich carbon support via the interaction with $\mathrm{O}$-containing groups. The excessive chelating agent bound to support surface will physically isolate the metal complex (secondary protection). The complex bound carbon is then mixed with melamine as a nitrogen source for subsequent pyrolysis to achieve M-NC SACs. During pyrolysis, the chelated metal complex can further secure metal atoms via the decomposed residues up to certain temperature $\left(\sim 500^{\circ} \mathrm{C}\right.$, Step 2$)$ (tertiary

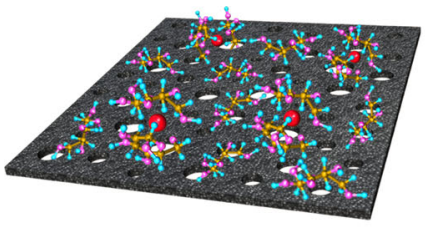

Chelation and isolation

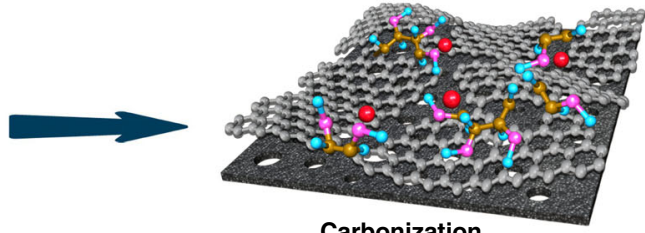

Carbonization

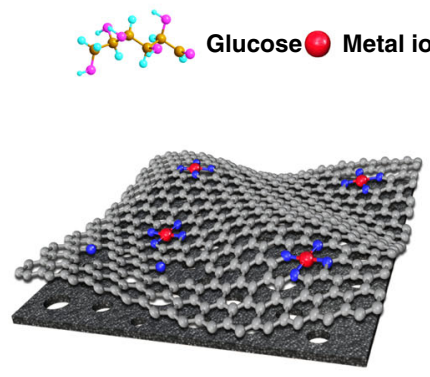

M-NC SAC

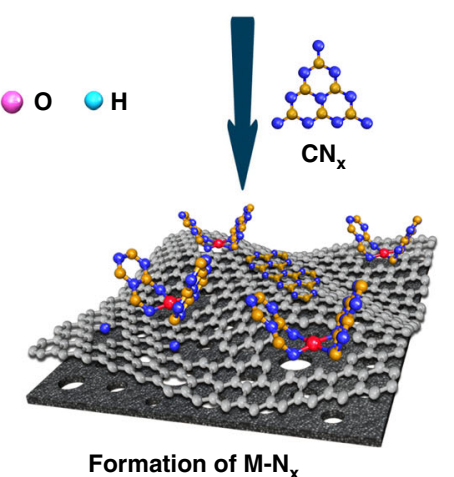

Fig. 1 The cascade anchoring strategy for the synthesis of M-NC SACs. First, chelating agent (glucose) efficiently sequesters metal ions and binds to O-rich carbon support, while excessive glucoses physically isolate glucose-metal complexes on carbon substrate. Second, the chelated metal complexes further secure metal atoms via the decomposed residues up to certain temperature. Third, $\mathrm{CN}_{\mathrm{x}}$ species decomposed from melamine at higher temperature subsequently capture metal atoms to form $M-N_{x}$ moieties and integrate into the pyrolyzed carbon layer 
protection), while carbon nitrogen species $\left(\mathrm{CN}_{\mathrm{x}}\right)$ (such as $\mathrm{C}_{3} \mathrm{~N}_{4}$ etc.) decomposed from melamine at higher temperature $\left(>\sim 600^{\circ} \mathrm{C}\right)$ can subsequently bind with metal atoms to form $\mathrm{M}-\mathrm{N}_{\mathrm{x}}$ moieties (Step 3), taking over the protection and preventing metal atoms from aggregation (quaternary protection). Systematical experiments reveal that such sequential protecting strategy allows for producing wide-ranging M-NC SACs with a high metal loading up to $12.1 \mathrm{wt} \%$, since the chelating interaction can take place between a wide range of metal ions and ligands ${ }^{46,47}$. Moreover, the carbon support can be low-cost commercial porous carbon; the chelating agents can be low-cost carbohydrates, such as glucose etc.; and the processing is very easy to scale up. In this regard, the present strategy is suitable for the low-cost mass production of M-NC SACs for diverse applications. As a demonstration, Fe-NC SAC shows a superior electrocatalytic activity for oxygen reduction reaction (ORR) in $0.1 \mathrm{M} \mathrm{KOH}$ with a half-wave potential of $0.90 \mathrm{~V}$ (all potentials are versus to RHE) and a kinetic mass current of $100.7 \mathrm{~A} \mathrm{~g}^{-1}$ at $0.9 \mathrm{~V}, 50 \mathrm{mV}$ and $65 \mathrm{~A} \mathrm{~g}^{-1}$ higher than that of state-of-the-art commercial $\mathrm{Pt} / \mathrm{C}$ catalyst, respectively. $\mathrm{Ni}-\mathrm{NC}$ SAC exhibits an excellent electrocatalytic activity for $\mathrm{CO}_{2}$ reduction to $\mathrm{CO}$ in terms of a good Faraday efficiency of $89 \%$ with a high current density of $30 \mathrm{~mA} \mathrm{~cm}^{-2}$ at $-0.85 \mathrm{~V}$.

\section{Results}

Synthesis and structural analysis of Fe-NC SAC. Since Fe-NC electrocatalysts are particularly interested for ORR to replace precious Pt-based commercial catalysts in fuel cells etc. We first take Fe-NC SAC as an example to demonstrate our strategy and its application. Fe-NC SAC was prepared in two steps. First, porous carbon (PC) support was ultrasonically dispersed in the solution containing $\mathrm{Fe}$ source and glucose (chelating agent). Second, the dried powder was ground with melamine (nitrogen source), followed by pyrolysis at $800^{\circ} \mathrm{C}$ to achieve Fe-NC SAC. The scanning electron microscope (SEM) image (Fig. 2a) shows that PC substrate (prepared by pyrolysis of potassium citrate as presented in the section of Methods) has a three-dimensional honeycomb-like morphology with plenty of macropores in several micrometers, which benefits mass transfer during both synthesis and application processes. X-ray diffraction (XRD) pattern (cyan curve in Fig. 2b) shows two typical broad peaks at 24.3 and $44.3^{\circ}$ for the PC substrate. The specific surface area and pore volume are measured to be $1713 \mathrm{~m}^{2} \mathrm{~g}^{-1}$ and $0.75 \mathrm{~cm}^{3} \mathrm{~g}^{-1}$, respectively (Supplementary Fig. 1). The pore size distribution analysis indicates most of nanopores centered at 1.3 and $2.0 \mathrm{~nm}$. $\mathrm{X}$-ray photoelectron spectroscopy (XPS) spectrum reveals plenty of O-species (10.05 at\%) on the surface of the PC substrate (Supplementary Fig. 2). X-ray energy dispersive spectroscopic (EDS)-mapping images show that the elemental $\mathrm{O}$ uniformly distributes on whole-carbon sheets (Supplementary Fig. 3). These features make such carbon substrate perfect for anchoring glucose-chelated Fe complex. The chelation of $\alpha$-D-glucose and $\mathrm{Fe}(\mathrm{III})$ ions to form $\mathrm{a}$-D-glucose-Fe(III) complex has been reported in the literatures ${ }^{47,48}$ and supported by our density functional theory (DFT) calculations (Supplementary Fig. 4). After mixing this PC substrate with glucose and Fe source, Fourier-transform infrared (FTIR) spectrum and EDS-mapping images indicate that glucose and glucose-chelated Fe complex cover on whole substrate in view of uniform FTIR signals of $\mathrm{O}-\mathrm{H}$ and distribution of elemental Fe and $\mathrm{O}$ (Supplementary Figs. 5 and 6). After pyrolysis at $800^{\circ} \mathrm{C}$ for $2 \mathrm{~h}$, no additional XRD peaks are detected except for those from carbon (Fig. 2b), implying no formation of crystallized Fe. Transmission electron microscopy (TEM) and high-resolution TEM (HRTEM) images (Fig. 2c, d) show that carbon sheet support is covered by flocculent sheet-like structures, which is a typical feature of pyrolyzed carbonaceous materials (glucose). Raman spectra (Supplementary Fig. 7) indicate that the $I_{\mathrm{D}} / I_{\mathrm{G}}$ ratio for $\mathrm{Fe}-\mathrm{NC} \mathrm{SAC}$ is slightly higher than carbon substrate (1.3 vs. 1.2 , PC substrate here went through the same pyrolysis for Raman recording), agreeing with the formation of the flocculent carbon layer in more disorder as observed in TEM image. No Fe-based particles, which are typically seen in pyrolyzed Fe-NC products, are found during TEM observation.
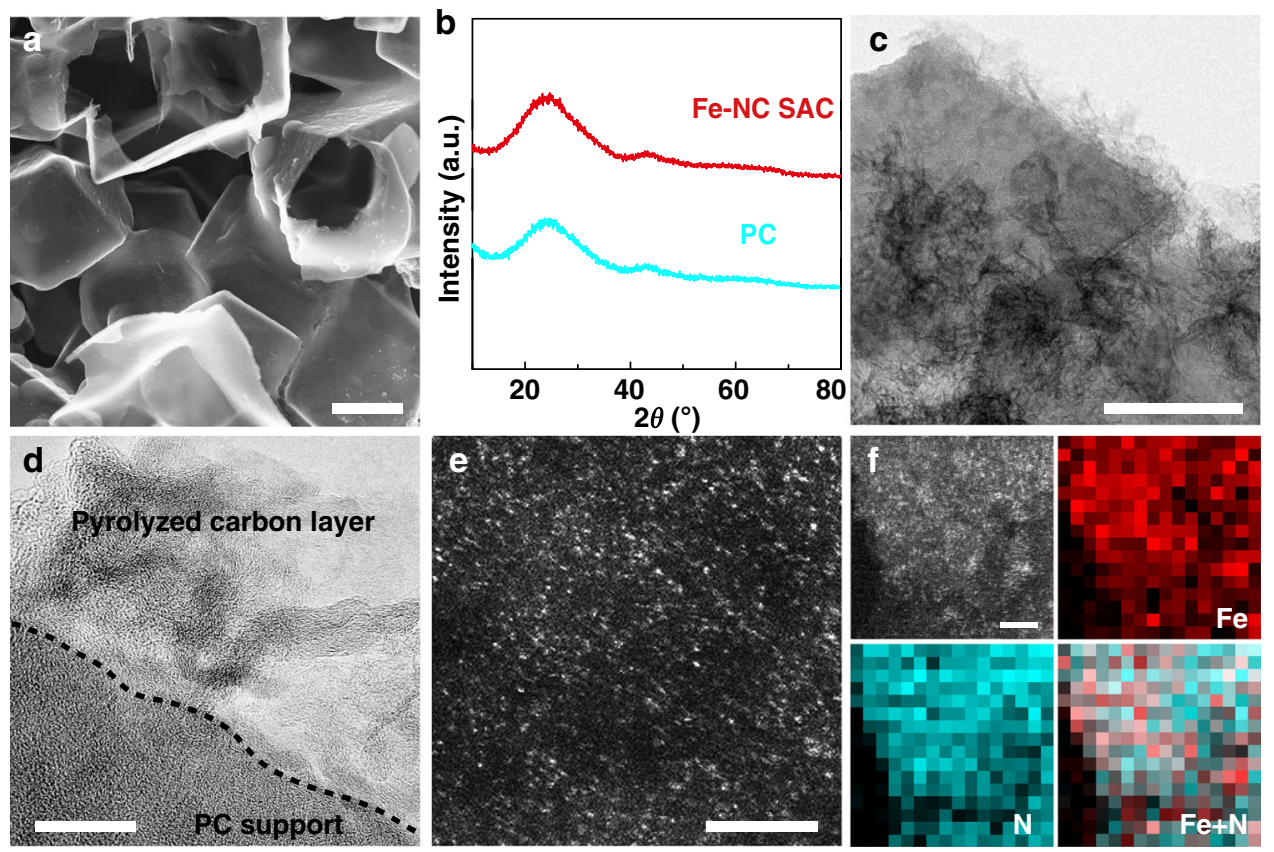

Fig. 2 Structural characterizations of PC support and Fe-NC SAC. a SEM image of PC support. b XRD patterns of PC and Fe-NC SAC. c TEM, d, HRTEM, e, HAADF-STEM images of Fe-NC SAC. $\mathbf{f}$ HAADF-STEM image and EELS mapping images of Fe, $\mathrm{N}$, and overlaid Fe and N on Fe-NC SAC. Scale bars, $1 \mu \mathrm{m}$ (a); $200 \mathrm{~nm}(\mathbf{c}) ; 20 \mathrm{~nm}(\mathbf{d}) ; 3 \mathrm{~nm}(\mathbf{e}) ; 2 \mathrm{~nm}(\mathbf{f})$ 
XRD and TEM results suggest that Fe may exist in a form of single atom. For clearly revealing the state of Fe, the high-angle annular dark-filed scanning TEM (HAADF-STEM) was used to acquire the evidence of $\mathrm{Fe}$ distribution at atomic resolution. As displayed in Fig. 2e and Supplementary Fig. 8, a number of bright spots in a single-atom size are clearly observed, which can be safely attributed to $\mathrm{Fe}$ atoms in this sample. The average size of spots is $1.04 \pm 0.35 \AA$ on a basis of statistical analysis on over 400 bright spots (inset in Supplementary Fig. 8a), corroborating these $\mathrm{Fe}$ atoms are mainly in single-atomic state. EDS spectrum evidences the existence of elemental $\mathrm{C}, \mathrm{Fe}$, and $\mathrm{N}$ (Supplementary Fig. 9a). Electron energy loss spectroscopy (EELS) and EDSmapping images depict the homogenous and uniform distribution of $\mathrm{Fe}$ and $\mathrm{N}$ in the Fe-NC SAC (Fig. $2 \mathrm{f}$ and Supplementary Figs. $9 \mathrm{~b}-\mathrm{f}$ and 10$)$.

Atomic structure analysis of Fe-NC SAC by XAFS and XPS. Element-selective X-ray absorption fine structure (XAFS) spectroscopy experiments were further conducted, including the extended X-ray absorption fine structure (EXAFS), which are powerful for determining the coordination environment and chemical state of absorbing centers with high sensitivity. As shown in Fig. 3a, X-ray absorption near-edge structure (XANES) spectrum and the first derivative XANES spectrum of $\mathrm{Fe}-\mathrm{NC}$ SAC are very similar to those of the reference iron phthalocyanine $(\mathrm{FePc})$ which has well-defined $\mathrm{Fe}-\mathrm{N}_{4}$ coordinated sites ${ }^{49-51}$, while distinct from those of the metallic Fe foil. This means Fe state in Fe-NC SAC should be similar to that in FePc. Figure 3b shows the Fourier transform (FT) $k^{3}$-weighted EXAFS spectra of Fe k-edge. Comparing with the Fe foil, no apparent peaks (2.20 and $4.42 \AA$ ) for $\mathrm{Fe}-\mathrm{Fe}$ coordination are observed in Fe-NC SAC. As expected, FT EXAFS spectrum of Fe-NC SAC has a strong peak at $1.50 \AA$. In reference to $\mathrm{FePc}$, this peak can be well assigned to $\mathrm{Fe}-\mathrm{N}$ distance where a nitrogen shell surrounds one $\mathrm{Fe}$ atom. Wavelet transform (WT) was also used to investigate the $\mathrm{Fe}$ K-edge EXAFS oscillations of Fe-NC SAC and the references. As shown in Fig. 3c, WT analysis of $\mathrm{Fe}-\mathrm{NC}$ SAC shows only one intensity maximum at about $4.5 \AA^{-1}$ for $\mathrm{Fe}-\mathrm{NC} \mathrm{SAC}$, which is very close to that in the reference FePc $\left(\sim 4.5 \AA^{-1}\right)$, but distinct from the feature of $\mathrm{Fe}$ foil $\left(7.0 \AA^{-1}\right)$. Combining with above HAADF-STEM results that all Fe species are atomically dispersed without detectable aggregation, these analyses suggest that $\mathrm{Fe}$ in $\mathrm{Fe}-\mathrm{NC}$ SAC exists in a similar state to the reference FePc. For giving further insights into the chemical configuration of $\mathrm{Fe}$, FT EXAFS fittings in $R, q$, and $k$ spaces were carried out to reveal the structural parameters and evaluate the fitting quality. As shown in Supplementary Figs. 11 and 12, all fittings are in good consistency with experimental data. The fitting results give an average coordination number of 4.3 for the first shell $(\mathrm{Fe}-\mathrm{N})$ and an average $\mathrm{Fe}-\mathrm{N}$ bond length of $1.99 \AA$ (see more details in Supplementary Table 1).

The chemical environments of $\mathrm{N}$ in $\mathrm{Fe}-\mathrm{NC}$ SAC and reference FePc were further investigated by near-edge XAFS (NEXAFS) and XPS technique. The N K-edge NEXAFS spectrum of Fe-NC SAC a

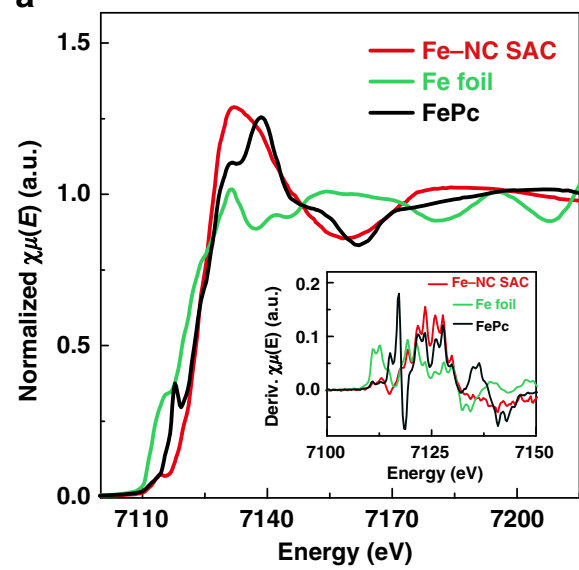

b

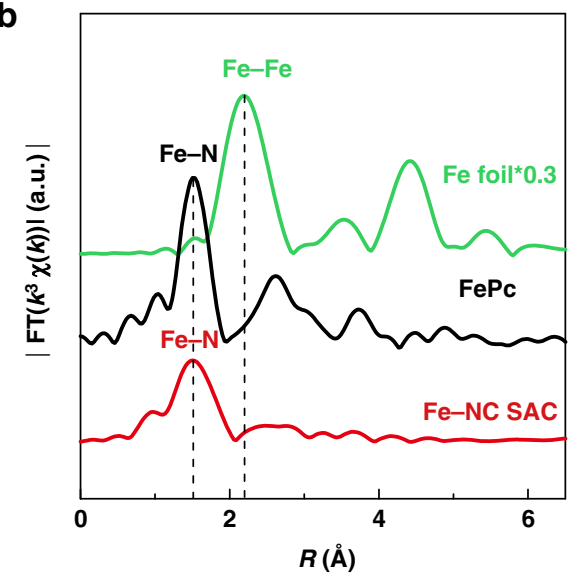

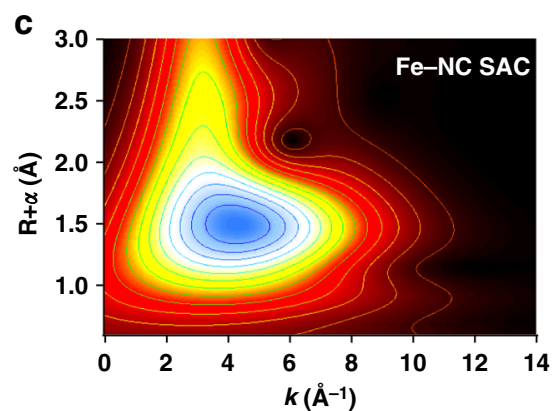
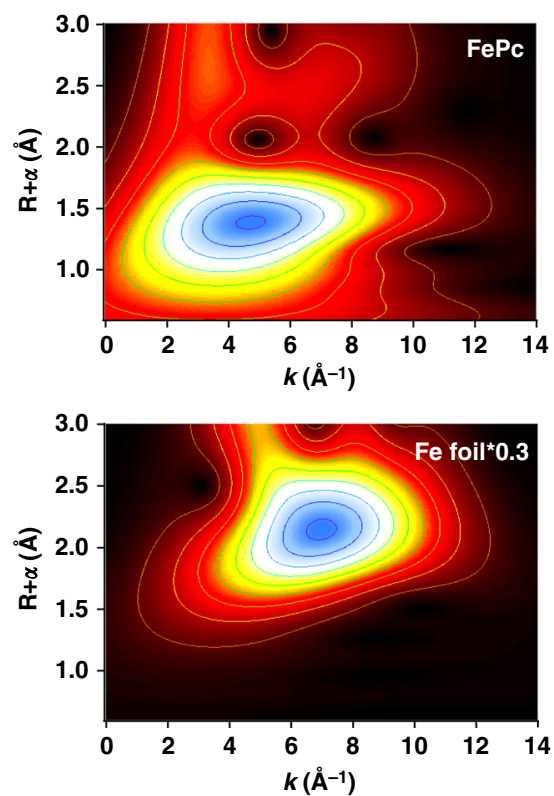

d
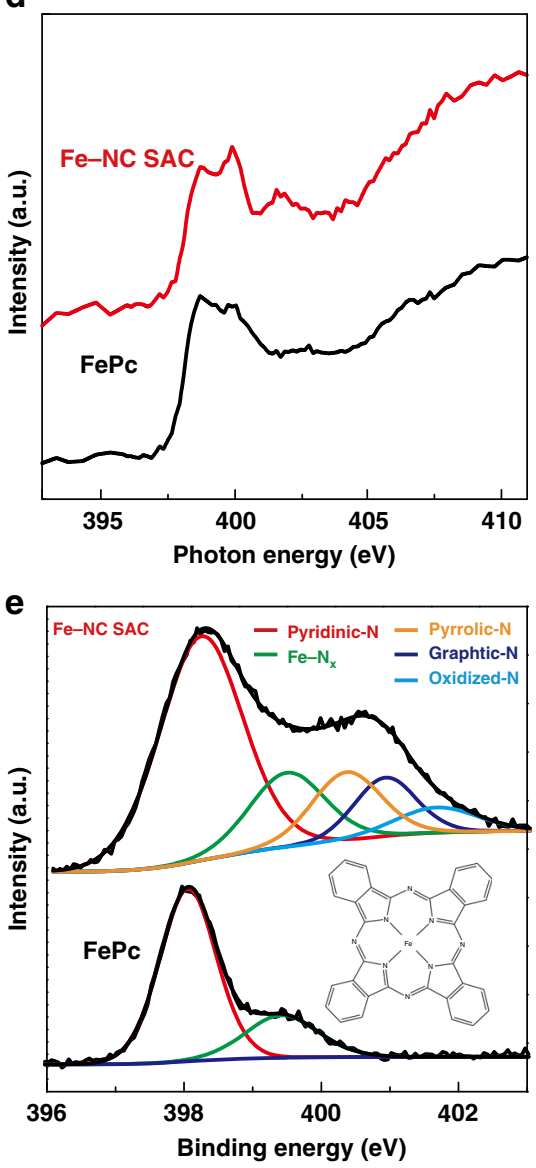

Fig. 3 Atomic structure analysis of Fe-NC SAC by XAFS and XPS. a Fe K-edge XANES spectra (inset: first-derivative curves), b Fourier transform of Fe K-edge EXAFS spectra, and $\mathbf{c}$, Wavelet transform of the $k^{3}$-weighted EXAFS data of Fe-NC SAC and reference samples (FePc and Fe foil). $\mathbf{d} \mathrm{N} K$-edge NEXAFS spectra and e, deconvoluted N 1s XPS spectra of Fe-NC SAC and reference FePc 
shows three distinct peaks at about 398.8, 399.8, and $401.8 \mathrm{eV}$ (Fig. 3d). The peak at $398.8 \mathrm{eV}$ can be assigned to pyridinic state or the state similar to aza-bridge in $\mathrm{FePc}$ due to the same energy position. The peak at $399.8 \mathrm{eV}$ shares the identical position with the $\mathrm{Fe}-\mathrm{N}$ bonding in $\mathrm{FePc}$, corroborating the existence of $\mathrm{Fe}-\mathrm{N}$ bonding in $\mathrm{Fe}-\mathrm{NC} \mathrm{SAC}^{52,53}$. The wide peak at $401.8 \mathrm{eV}$ could be assigned to pyrrolic or other nitrogen states according to the literatures ${ }^{54,55}$. For XPS spectra shown in Fig. 3e, the N $1 s$ signal for Fe-NC SAC can be deconvoluted into several characteristic peaks. The XPS signals at 398.3, 399.5, 400.4, 400.9, and $401.7 \mathrm{eV}$ are assigned to pyridinic- $\mathrm{N}, \mathrm{Fe}-\mathrm{N}_{\mathrm{x}}$, pyrrolic- $\mathrm{N}$, graphitic- $\mathrm{N}$, and oxidized-N, respectively ${ }^{56-58}$. The peak at $399.5 \mathrm{eV}$ indicates the presence of $\mathrm{N}$ in the chemical state similar to $\mathrm{Fe}-\mathrm{N}_{\mathrm{x}}$ moiety in FePc. ${ }^{59,60}$ The high-resolution Fe 2p XPS spectrum (Supplementary Fig. 13) indicates that Fe exists in form of iron (II). No clear signal of metallic $\mathrm{Fe}$ is detected. These XPS data agree with the existence of $\mathrm{Fe}-\mathrm{N}_{\mathrm{x}}$ coordination in $\mathrm{Fe}-\mathrm{NC}$ SAC. Moreover, the NEXAFS spectra of O K-edge (Supplementary Fig. 14) for Fe-NC SAC shows no distinct features of O-Fe bonding which should appear in the region from 529 to $531 \mathrm{eV}^{61}$, suggesting there is no significant $\mathrm{O}-\mathrm{Fe}$ bonding in our $\mathrm{Fe}-\mathrm{NC}$ SAC. All the above analyses support that $\mathrm{Fe}-\mathrm{N}$ bonding is the dominated $\mathrm{Fe}$ state in $\mathrm{Fe}-\mathrm{NC}$ SAC. The amount of $\mathrm{Fe}$ in $\mathrm{Fe}-\mathrm{NC}$ SAC is further determined by thermogravimetric analysis (TGA) (Supplementary Fig. 15). The Fe loading of $8.9 \mathrm{wt} \%$ is significantly higher than those in reported SACs (Supplementary Table 2), suggesting the present strategy is able to deliver single-atomic catalysts with a high metal loading. Importantly, it should be noted that this cascade-anchoring strategy can achieve the mass production of $\mathrm{Fe}-\mathrm{NC}$ SAC due to facile and manageable processing. As a demonstration, about $8 \mathrm{~g}$ of $\mathrm{Fe}-\mathrm{NC}$ SAC is easily obtained in a one-batch synthesis (Supplementary Fig. 16) in the laboratory. XRD pattern and TEM images indicate that the product shares the similar structure to the sample characterized above (Supplementary Fig. 17).

Insight into the formation process of Fe-NC SAC. As mentioned above, the present cascade-anchoring strategy is able to prepare single-atomic $\mathrm{Fe}-\mathrm{NC}$ materials with a high Fe loading up to $8.9 \mathrm{wt} \%$. It is found that the successive protection tactics at each stage during the synthesis are essential to prevent the aggregation of $\mathrm{Fe}$ atoms at high loading condition. (1) Glucose chelating effect: The chelation of $\mathrm{Fe}$ ion with glucose is the first protection step to well isolate $\mathrm{Fe}$ ion in physical space. The control sample (Fe@C-N) prepared in parallel, except for no addition of glucose, shows clearly iron/iron carbide nanoparticles on carbon sheets with some nanotubes formed via Fe-catalyzed growth during the decomposition of melamine, as evidenced by XRD pattern (Fig. 4a) and TEM images (Fig. 4b and Supplementary Fig. 18). The crystallized Fe species are even observed in the sample prepared at $500^{\circ} \mathrm{C}$, without addition of glucose (XRD pattern in Supplementary Fig. 19 for control sample Fe@C-N-500). The role of chelation is corroborated by another two control experiments. If inorganic iron salt (iron (III) nitrate) is substituted with iron (III) acetylacetonate $\left(\mathrm{Fe}(\mathrm{acac})_{3}\right)$, where the strong interaction between Fe ion and acetylacetone excludes the chelation of glucose and $\mathrm{Fe}$ ion, plenty of iron carbide nanoparticles are formed instead of isolated $\mathrm{Fe}$ atoms as suggested by XRD (Fig. 4a) and TEM results (Fig. 4c and Supplementary Fig. 20). Moreover, if other chelating agent such as ethylenediamine tetraacetic acid (EDTA) is used to replace glucose, XRD, TEM, and EDS-mapping results suggest that the similar Fe-NC SAC material is obtained (Fig. 4a, d and Supplementary Figs. 21 and 22). In view of the low cost, availability and solubility in water for mass production, glucose was used as chelating agents in our experiments. (2) Physical isolation of complex: The physical isolation of Fe centers is also important. The excessive amount of glucose is found to be necessary for achieving single-atomic $\mathrm{Fe}-\mathrm{N}_{\mathrm{x}}$ in the final product. The insufficient amount of glucose (such as 5:1 molar ratio of glucose: $\mathrm{Fe}$ ) cannot keep Fe atoms away enough to prevent their aggregation during high-temperature pyrolysis so that some Fe-based nanoparticles are produced (Fe-NC-Low Glu, Supplementary Figs. 23 and 24). (3) Carbon substrate: O-rich substrate with a high surface area is critical for the synthesis of high-loading single-atomic $\mathrm{Fe}-\mathrm{N}_{\mathrm{x}}$. Commercial Ketjenblack (KB) with a comparable surface
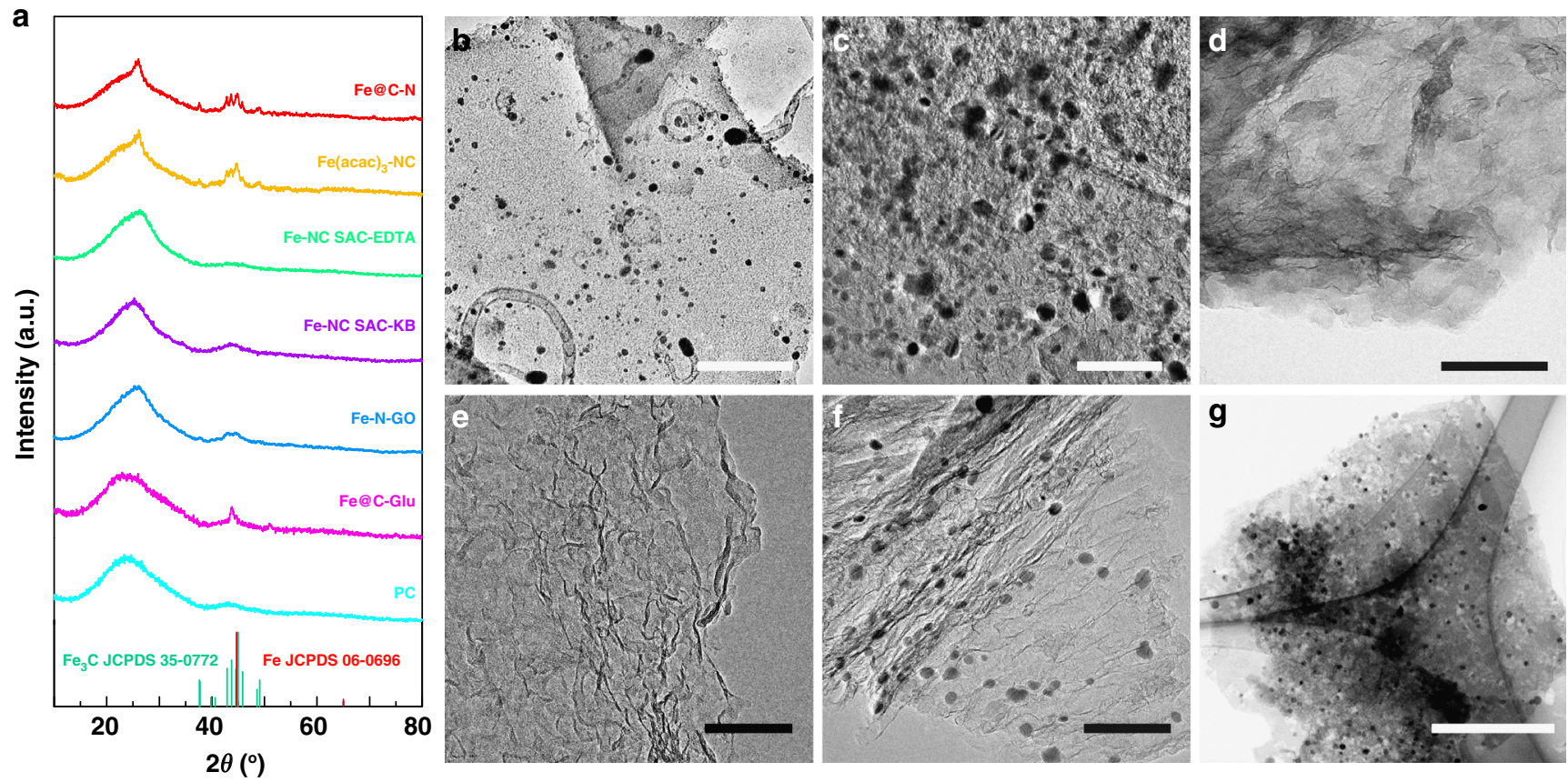

Fig. 4 Structural characterizations of control samples. a XRD patterns and b-g, TEM images of Fe@C-N (b), Fe(acac) 3 -NC (c), Fe-NC SAC-EDTA (d), Fe-NC SAC-KB (e), Fe-N-GO (f), and Fe@C-Glu (g). Scale bars: 200 nm (b, d); 100 nm (c, e, f); 500 nm (g) 
area of $1400 \mathrm{~m}^{2} \mathrm{~g}^{-1}$ can be used to replace PC support (Supplementary Fig. 25). XRD pattern in Fig. 4a and TEM images in Fig. $4 \mathrm{e}$ and Supplementary Fig. 26 imply that the similar catalyst structure is obtained. It is noted that the acid pre-treatment of $\mathrm{KB}$ to create O-rich surface is necessary to achieve uniform distribution of glucose-Fe complex and physically isolate Fe centers on substrate. Without acid treatment, $\mathrm{KB}$ is not dispersed well in aqueous solution and $\mathrm{Fe}$ aggregates are obtained. In contrast, if graphene oxide (GO) with a low surface area of $90 \mathrm{~m}^{2} \mathrm{~g}^{-1}$ is used (Supplementary Fig. 25), a number of Fe-based nanoparticles are produced as evidenced by XRD pattern (Fig. 4a) and TEM images (Fig. 4f and Supplementary Fig. 27). It is believed that such surface area is not sufficient to isolate glucose-Fe complex, leading to the aggregation of active $\mathrm{Fe}$ atoms during pyrolysis. (4) Cascade protection at different stages: As mentioned above, excessive glucose to chelate $\mathrm{Fe}$ ion and physically isolate them is required to achieve $\mathrm{Fe}-\mathrm{NC}$ SAC; however, it is not sufficient. It is found that the glucose protection is only effective at a moderate temperature. The control sample prepared without addition of melamine shows no crystalline $\mathrm{Fe}$ species at the pyrolysis temperature up to $500{ }^{\circ} \mathrm{C}$ (Fe@C-Glu-500) (Supplementary Fig. 19), whereas increasing the pyrolysis temperature to $600^{\circ} \mathrm{C}$ crystallized $\mathrm{Fe}_{2} \mathrm{O}_{3}$ phase can be clearly detected in XRD pattern (Fe@CGlu-600) (Supplementary Fig. 19). Further increasing the temperature to $800^{\circ} \mathrm{C}$ metallic Fe-based phase shows up in XRD pattern (Fig. 4a) of the sample (Fe@C-Glu) instead of $\mathrm{Fe}_{2} \mathrm{O}_{3}$ phase due to carbothermal reduction and a number of nanoparticles can be clearly identified in TEM images (Fig. 4g and Supplementary Fig. 28). Without melamine these nanoparticles can even migrate, leaving the holes in carbon substrate. It is known that melamine decomposes to $\mathrm{N}$-containing species such as $\mathrm{C}_{3} \mathrm{~N}_{4}$ etc. at over $400^{\circ} \mathrm{C}^{62}$. In the synthesis of $\mathrm{Fe}-\mathrm{NC} \mathrm{SAC}$, when the $\mathrm{Fe}$-glucose complexes start to decompose and release $\mathrm{Fe}$ atoms at elevated temperature, the surrounding abundant active $\mathrm{CN}_{\mathrm{x}}$ species decomposed from excessive amount of melamine will instantly capture these active $\mathrm{Fe}$ atoms by forming $\mathrm{Fe}-\mathrm{N}$ bonding as $\mathrm{Fe}-\mathrm{N}_{\mathrm{x}}$ species since it is an energy-favorable process, similar to the case of the formation of $\mathrm{Pd}-\mathrm{N}^{63}$. These $\mathrm{Fe}-\mathrm{N}_{\mathrm{x}}$ species will be subsequently incorporated into the carbon network newly evolved from the graphitization of glucose and melamine during pyrolysis, preventing them from aggregation. The single-atomically dispersed $\mathrm{Fe}-\mathrm{N}_{\mathrm{x}}$ sites have been evidenced by the above-mentioned analyses.

Electrocatalytic performance evaluation of Fe-NC SAC. As a demonstration of the potential applications of M-NC SACs, the electrocatalytic activity of Fe-NC SAC for ORR was evaluated and compared with benchmark $\mathrm{Pt} / \mathrm{C}$ catalyst and control samples (Fe@C-N, Fe@C-Glu, and C-N-Glu). As shown in Fig. 5a and Supplementary Table 3, the state-of-the-art commercial Pt/C (20 wt\%) exhibits a good ORR activity in terms of an onset potential (defined by the potential at which the current density reaches $\left.0.1 \mathrm{~mA} \mathrm{~cm}^{-2}\right)^{64,65}$ of $0.96 \mathrm{~V}$ and a half-wave potential of $0.85 \mathrm{~V}$. While the present Fe-NC SAC obviously shows a a
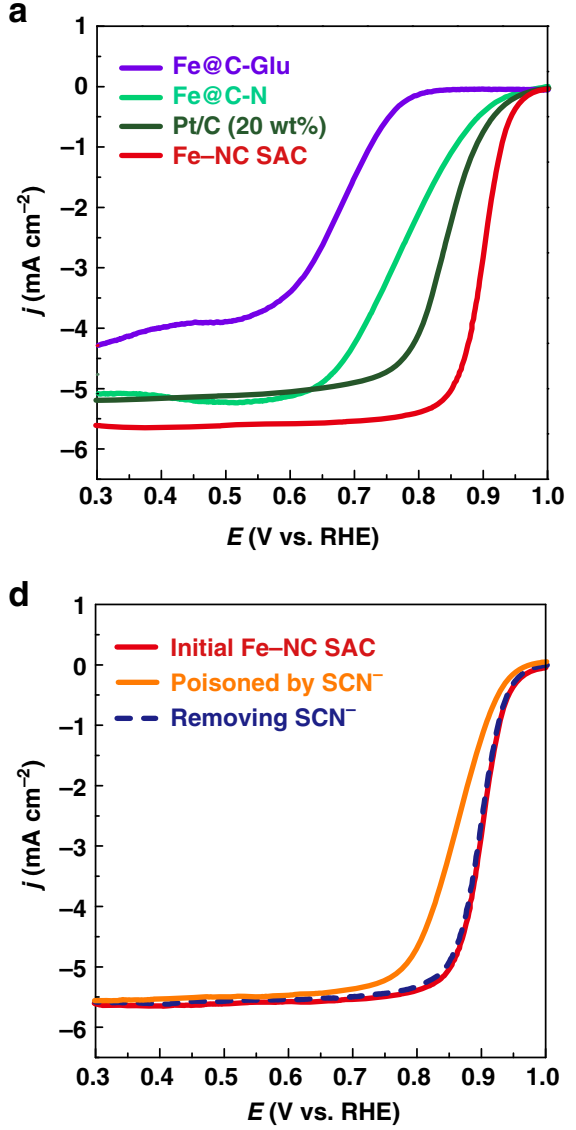

b

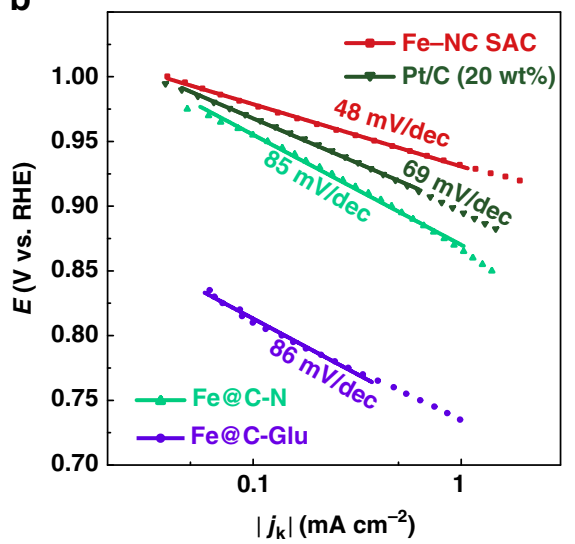

e

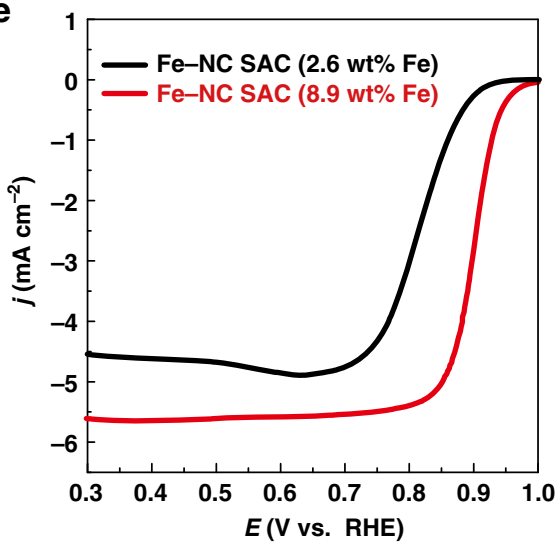

C

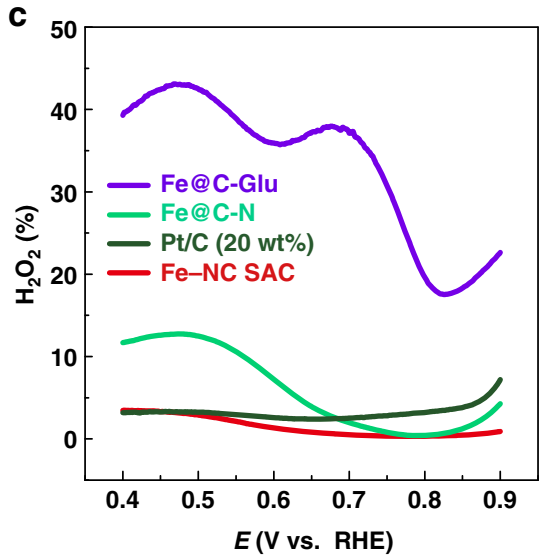

$\mathbf{f}$

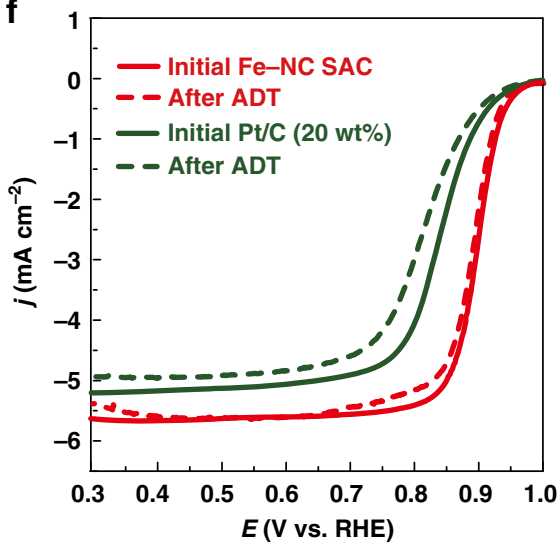

Fig. 5 Evaluation of electrocatalytic performance of Fe-NC SAC for ORR. a Steady-state ORR polarization curves of Fe-NC SAC, Pt/C and control samples (Fe@C-N and Fe@C-Glu).b Corresponding Tafel plots. c Hydrogen peroxide yield. d Steady-state ORR polarization curves of Fe-NC SAC recorded in $\mathrm{O}_{2-}$ saturated $0.1 \mathrm{M} \mathrm{KOH}$ with or without poisoning by $0.01 \mathrm{M} \mathrm{SCN}^{-}$, and one collected after removing SCN ${ }^{-}$. e Steady-state ORR polarization curves of Fe-NC $\mathrm{SAC}$ with different Fe loading. $\mathbf{f}$ Steady-state ORR polarization curves of Fe-NC SAC and Pt/C before and after 5000 potential scanning cycles in $\mathrm{O}_{2-}$ saturated $0.1 \mathrm{M} \mathrm{KOH}$ 
positively shifted onset potential $(0.98 \mathrm{~V})$ and a half-wave potential $(0.90 \mathrm{~V})$, which are 20 and $50 \mathrm{mV}$ more positive than those for $\mathrm{Pt} / \mathrm{C}$ catalyst and superior to most of non-precious metal ORR electrocatalysts (Supplementary Table 4). The mass activity of Fe-NC-SAC is calculated to be $9.0 \mathrm{Ag}^{-1}$ at $0.90 \mathrm{~V}$. What is more, the Fe-NC SAC shows a high kinetic mass current of $100.7 \mathrm{~A} \mathrm{~g}^{-1}$ at $0.90 \mathrm{~V}, 65 \mathrm{~A} \mathrm{~g}^{-1}$ larger than $\mathrm{Pt} / \mathrm{C}\left(35.7 \mathrm{~A} \mathrm{~g}^{-1}\right)$. In contrast, control sample Fe@C-N (prepared without glucose), Fe@C-Glu (prepared without melamine), and C-N-Glu (prepared without iron) demonstrate poor ORR electrocatalytic activities (Supplementary Fig. 29). The superior ORR electrocatalytic activity of $\mathrm{Fe}-\mathrm{NC} \mathrm{SAC}$ is further confirmed by the smallest Tafel slope (48 vs. $69 \mathrm{mV} \mathrm{dec}^{-1}$ for Pt/C) (Fig. 5b) and the lowest $\mathrm{H}_{2} \mathrm{O}_{2}$ yield (below $3.5 \%$ at the whole potential range) (Fig. 5c). These results indicate that ORR process on Fe-NC SAC follows a four-electron pathway with a high electrocatalytic efficiency for ORR.

To get insight into the active sites for ORR in Fe-NC SAC, additional control experiments were carried out. Although there is still debate about the most active sites for ORR in $\mathrm{Fe}-\mathrm{N}-\mathrm{C}$ catalysts due to complicate catalyst structures, $\mathrm{Fe}-\mathrm{N}_{\mathrm{x}}$ site is generally considered to play an important role in catalyzing ORR. It has been reported that $\mathrm{SCN}^{-}$ion is able to strongly interact with $\mathrm{Fe}$ center, thus poisoning $\mathrm{Fe}-\mathrm{N}_{\mathrm{x}}$ coordination site ${ }^{66}$. Since the coordination of Fe-SCN is stable in the acidic condition but not in the alkaline medium, we first test the ORR activity of $\mathrm{Fe}-\mathrm{NC}$ SAC in $\mathrm{O}_{2}$-saturated $0.1 \mathrm{M} \mathrm{HClO}_{4}$ containing $0.01 \mathrm{M}$ $\mathrm{SCN}^{-}$. The result shows that the half-wave potential decrease distinctly by about $116 \mathrm{mV}$ comparing with the curve measured without addition of $0.01 \mathrm{M} \mathrm{SCN}^{-}$(Supplementary Fig. 30), which can be ascribed to the blocking of $\mathrm{Fe}-\mathrm{N}_{\mathrm{x}}$ active sites by $\mathrm{SCN}^{-}$. Moreover, when rinsing this pre-poisoned electrode to $\mathrm{pH}=7$ and re-testing it in $\mathrm{O}_{2}$-saturated $0.1 \mathrm{M} \mathrm{KOH}$, the half-wave potential negatively shifts by about $30 \mathrm{mV}$ compared with the values measured in $0.1 \mathrm{M} \mathrm{KOH}$ without $\mathrm{SCN}^{-}$(Fig. $5 \mathrm{~d}$ ) ${ }^{67,68}$. It is noted that polarization curve can gradually recover to its original state during the measurements since the blocked $\mathrm{Fe}-\mathrm{N}_{\mathrm{x}}$ coordination sites can be gradually released as the dissociation of $\mathrm{Fe}-\mathrm{SCN}^{-}$in $\mathrm{KOH}$ (Fig. $5 \mathrm{~d}$ ). These results suggest that $\mathrm{Fe}-\mathrm{N}_{\mathrm{x}}$ sites should be the most active sites in $\mathrm{Fe}-\mathrm{NC}$ SAC in view that only two possible active sites exist, i.e. $\mathrm{Fe}-\mathrm{N}_{\mathrm{x}}$ sites and $\mathrm{N}$-doped carbon. To further demonstrate the role of $\mathrm{Fe}-\mathrm{N}_{\mathrm{x}}$ sites, we prepared another control sample $\mathrm{Fe}-\mathrm{NC} \mathrm{SAC}$ with a low $\mathrm{Fe}$ loading via reducing the amount of Fe source and keeping all other conditions same. The characterizations indicate that the similar catalyst to $\mathrm{Fe}-\mathrm{NC}$ SAC was achieved except for the low $\mathrm{Fe}$ loading of $2.6 \mathrm{wt} \%$ (vs. $8.9 \mathrm{wt} \%$ for Fe-NC SAC) (Supplementary Fig. 31). The electrochemical measurements show that the ORR electrocatalytic activity is significantly attenuated in terms of 80 $\mathrm{mV}$ negatively shifted half-wave potential and decreased limiting current density (Fig. 5e). This result corroborates that $\mathrm{Fe}-\mathrm{N}_{\mathrm{x}}$ sites in $\mathrm{Fe}-\mathrm{NC}$ SAC are the efficient active sites for delivering a high ORR activity and the loading of $\mathrm{Fe}-\mathrm{N}_{\mathrm{x}}$ sites affects the activity. Furthermore, it is noted that $\mathrm{Fe}-\mathrm{NC}$ SAC also shows a better ORR electrocatalytic activity than the reference sample FePc/C which was prepared by loading $\mathrm{FePc}$ on carbon substrate with a similar Fe loading (see more details in Supplementary Fig. 32). This could be ascribed to the severe aggregation of FePc and the possible differences in the environments of $\mathrm{Fe}-\mathrm{N}_{\mathrm{x}}$ centers for our $\mathrm{Fe}-\mathrm{NC}$ SAC and reference $\mathrm{FePc} / \mathrm{C}$.

Durability is another important criterion for assessing electrocatalyst performance. The durability of Fe-NC SAC was evaluated using accelerated durability test (ADT) by cyclic voltammetry experiment from 0.6 to $1.0 \mathrm{~V}$ at $50 \mathrm{mV} \mathrm{s}^{-1}$ in $\mathrm{O}_{2}$ saturated $0.1 \mathrm{M} \mathrm{KOH}$. As shown in Fig. 5f, polarization curves recorded after 5000 cycles for Fe-NC SAC display a negligible degradation for half-wave potential and limiting current density, indicating its superior durability in alkaline medium. TEM and EDS-mapping images reveal that no Fe aggregation is observed, and the atomic dispersion of $\mathrm{Fe}-\mathrm{N}_{\mathrm{x}}$ sites retains in Fe-NC SAC after ADT (Supplementary Fig. 33). By contrast, the commercial Pt/C catalyst shows a $20 \mathrm{mV}$ loss in half-wave potential and appreciable reduction of limiting current density after ADT. The significant activity degradation for $\mathrm{Pt} / \mathrm{C}$ catalyst should be attributed to the serious dissolution/agglomeration of Pt nanoparticles during ADT, as shown in Supplementary Figs. 34 and $35^{69}$.

Demonstration for general synthesis of M-NC SACs. Inspired by the simplicity and general applicability of the present cascadeprotection strategy, we easily extend it to prepare a wide range of other $\mathrm{M}$-NC SACs $(\mathrm{M}=\mathrm{Mn}, \mathrm{Co}, \mathrm{Ni}, \mathrm{Cu}, \mathrm{Mo}, \mathrm{Pt}$, etc.) with a high metal loading. As shown in Fig. 6a-f and Supplementary Fig. 36, the white spots in a size of single atom in HAADF-STEM images indicate that $\mathrm{Mn}, \mathrm{Co}, \mathrm{Ni}, \mathrm{Cu}, \mathrm{Mo}$, and $\mathrm{Pt}$ are singleatomically dispersed in the PC substrate. A couple of spots with different size could be ascribed to the metal atom imaged at different focusing planes or possible atom cluster. XRD patterns prove that no crystallized metal-based phases are detected (Supplementary Fig. 37). EDS spectra, corresponding mapping images and EELS-mapping images confirm the existence of element metal and nitrogen as well as their homogenous distribution (Supplementary Figs. 38-45). These results suggest that these $\mathrm{M}-\mathrm{NC}$ SACs should share the similar structure to Fe-NC SAC. TGA analyses give the metal loading from 12.1 to $4.5 \mathrm{wt} \%$ (Fig. $6 \mathrm{~g}$ and Supplementary Fig. 46). The variation could be ascribed to the differences in the interaction of glucose and metal ions as well as the carbon loss in the presence of different metals. The exploration for the applications of these M-NC SACs are expected in view of the high density of single-atomically dispersed metal- $\mathrm{N}_{\mathrm{x}}$ active centers. For example, Ni-NC SAC exhibits the potential for $\mathrm{CO}_{2}$ reduction to $\mathrm{CO}$ and shows an $89 \%$ of Faraday efficiency at $-0.85 \mathrm{~V}$ with a $30 \mathrm{~mA} \mathrm{~cm}^{-2}$ of current density for CO (Supplementary Fig. 47 and Fig. 6h, i). This high current density outperforms most reported Ni-based singleatom catalysts, which should be attributed to the high-density Ni-based active sites with a Ni loading of $5.9 \mathrm{wt} \%$ in Ni-NC SAC (Supplementary Tables 5 and 6).

\section{Discussion}

In summary, a cascade-protection strategy is developed to synthesize single-atomic metal- $\mathrm{N}_{\mathrm{x}}$ sites on $\mathrm{N}$-doped carbon with a high metal loading up to $12.1 \mathrm{wt} \%$. The single-atomic dispersion of metal atoms and the formation of metal- $\mathrm{N}_{\mathrm{x}}$ sites are evidenced by the different analytic techniques, including HAADF-STEM, EELS, XANES, EXAFS, and NEXAFS etc. The formation process of single-atomic metal- $\mathrm{N}_{\mathrm{x}}$ sites and the effectiveness of the cascade-protection strategy are systematically investigated by a series of control experiments. The results suggest that the chelation of glucose and $\mathrm{Fe}$ ion, physical isolation of glucose-Fe complex via excessive glucose, support with sufficient $\mathrm{O}$-species and surface area to anchor and isolate glucose-Fe complex, and the binding with $\mathrm{N}$-species at elevated pyrolysis temperature are required to achieve the high-loading single-atomic $\mathrm{Fe}-\mathrm{N}_{\mathrm{x}}$ sites. As a demonstration, the electrocatalytic performance of $\mathrm{Fe}-\mathrm{NC}$ SAC for ORR is evaluated. Benefiting from the high-loading $\mathrm{Fe}-\mathrm{N}_{\mathrm{x}}$ active sites and their single-atomic dispersion for exposing each site, $\mathrm{Fe}-\mathrm{NC}$ SAC exhibits a superior ORR electrocatalytic activity and durability with a half-wave potential of $0.90 \mathrm{~V}$ and a kinetic mass current of $100.7 \mathrm{~A} \mathrm{~g}^{-1}$ at $0.90 \mathrm{~V}, 50 \mathrm{mV}$ and $65 \mathrm{~A} \mathrm{~g}^{-1}$ higher than state-of-the-art $\mathrm{Pt} / \mathrm{C}$, respectively. The poisoning and 

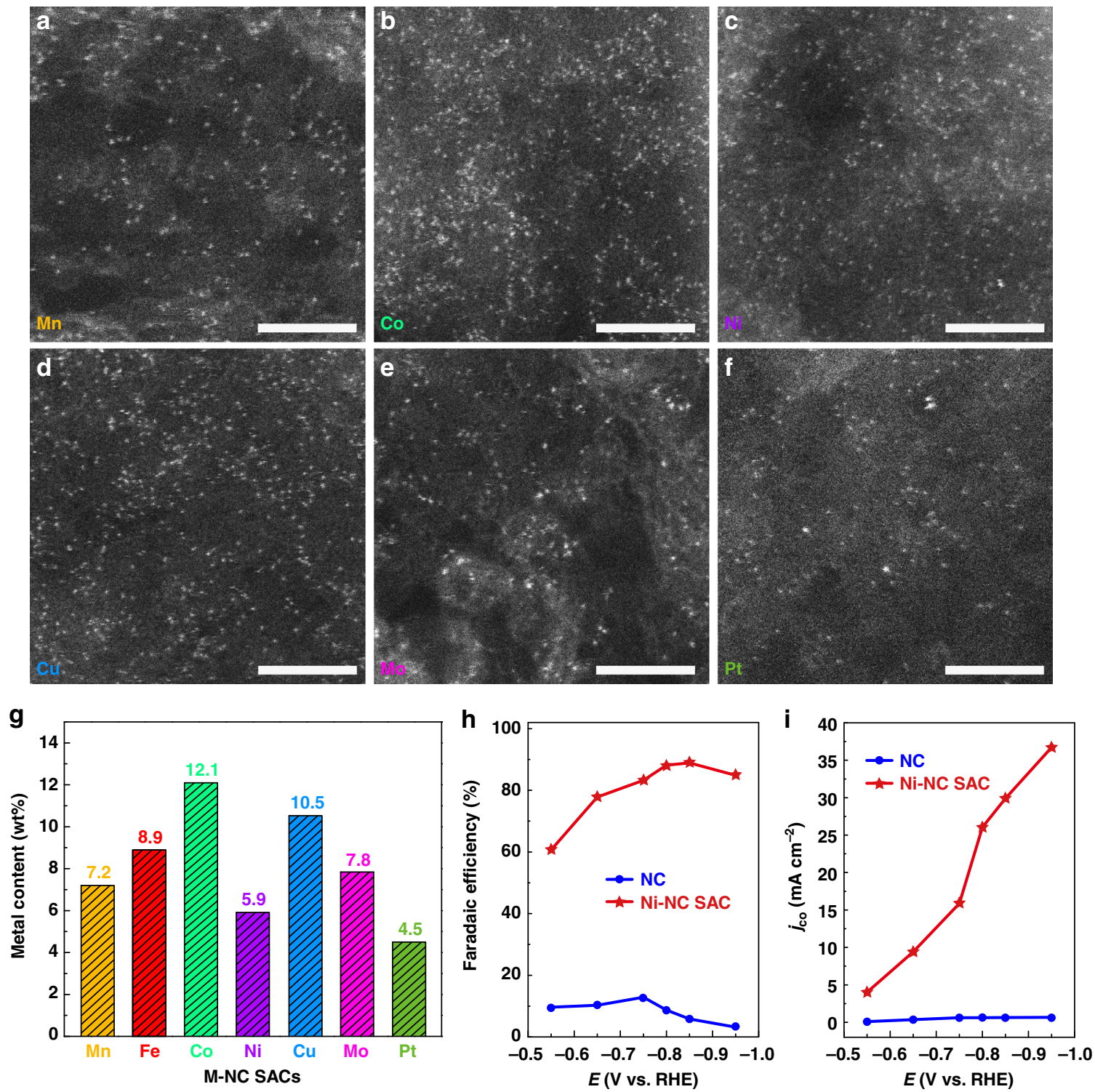

Fig. 6 Atomic structure characterizations and loading analysis of M-NC SACs. a-f HAADF-STEM images for Mn-NC SAC (a), Co-NC SAC (b), Ni-NC SAC (c), Cu-NC SAC (d), Mo-NC SAC (e), and Pt-NC SAC (f). Scale bars, $3 \mathrm{~nm}$ (a-f). $\mathbf{g}$ Metal loading in M-NC SACs. h Faradaic efficiency of CO, and $\mathbf{i}$, $j_{\mathrm{co}}$ for Ni-NC SAC and control sample (NC)

loading-dependent experiments indicate that such ORR performance should be from $\mathrm{Fe}-\mathrm{N}_{\mathrm{x}}$ sites. The mass production and scalability of the present strategy is demonstrated by synthesis over $8 \mathrm{~g}$ of such Fe-NC SAC with consistent ORR activity in a single-lab batch. The universal syntheses of other M-NC SACs $(\mathrm{M}=\mathrm{Mn}$, Co, Ni, Cu, Mo, Pt, etc.) demonstrate that such strategy can be easily adapted to apply different chelating agents, substrates, and metal sources for a wide range of metal-NC SACs. In view of high-loading metal- $\mathrm{N}_{\mathrm{x}}$ coordination sites in singleatomic level, these materials are expected for diverse applications, including electrocatalysis and heterogeneous catalysis etc.

\section{Methods}

Synthesis of PC support. In total, $8 \mathrm{mmol}$ of potassium citrate (Alfa Aesar Co., Ltd.) was pyrolyzed at $800{ }^{\circ} \mathrm{C}$ for $1 \mathrm{~h}$ in a tube furnace and $\mathrm{Ar}$ atmosphere. The black solid product was washed with $\mathrm{H}_{2} \mathrm{SO}_{4}$ solution $(0.5 \mathrm{M}$ ) (Alfa Aesar Co., Ltd.) and water $(18.2 \mathrm{M} \Omega)$ to remove inorganic impurities. After drying at $60^{\circ} \mathrm{C}$, the $\mathrm{PC}$ support was achieved.

Synthesis of Fe-NC SAC. In total, $60 \mathrm{mg}$ of PC, $0.3 \mathrm{mmol}$ of iron (III) nitrate nonahydrate (Alfa Aesar Co., Ltd.), and $6.7 \mathrm{mmol}$ of $\alpha$-D-glucose (Sinopharm
Chemical Reagent Co., Ltd.) were dispersed in $5 \mathrm{~mL}$ of ultrapure water, and sonicated for $30 \mathrm{~min}$ to get a homogenous black suspension. The slurries were harvested after washing with water and drying at $60^{\circ} \mathrm{C}$, and then grounded together with melamine (Alfa Aesar Co., Ltd.) at a mass ratio of 1:5. The obtained powder was placed into a tube furnace and heated to $800^{\circ} \mathrm{C}$ under Ar flow $(100 \mathrm{sccm})$. After $2 \mathrm{~h}$ of pyrolysis, the black Fe-NC SAC was obtained. M-NC SACs $(\mathrm{M}=\mathrm{Mn}, \mathrm{Co}, \mathrm{Ni}, \mathrm{Cu}, \mathrm{Mo}$, and $\mathrm{Pt})$ were prepared via the same procedures, except for using manganese nitrate hexahydrate (Alfa Aesar Co., Ltd.), cobalt nitrate hexahydrate (Alfa Aesar Co., Ltd.), nickel nitrate hexahydrate (Alfa Aesar Co., Ltd.), cupric nitrate hemipentahydrate (Alfa Aesar Co., Ltd.), ammonium molybdate tetrahydrate (Alfa Aesar Co., Ltd.), and (hydro)chloroplatinic acid (Alfa Aesar Co., Ltd.) as the metal precursor, respectively.

Synthesis of control samples. (1) Fe-NC SAC-EDTA was synthesized in parallel by the same method as that for Fe-NC SAC, except for using the same mass amount of EDTA (Alfa Aesar Co., Ltd.) instead of glucose. Fe(acac) $)_{3}-\mathrm{NC}$ was prepared in parallel by the same method as that for Fe-NC SAC, except for using the same molar amount of $\mathrm{Fe}(\mathrm{acac})_{3}$ (Alfa Aesar Co., Ltd.) instead of iron (III) nitrate nonahydrate. (2) Fe-NC SAC-KB and Fe-N-GO were prepared in parallel by the same method as that $\mathrm{Fe}-\mathrm{NC}$ SAC except for using the same mass amount of commercial KB $\left(1400 \mathrm{~m}^{2} \mathrm{~g}^{-1}\right)$ (Ketjenblack EC-600JD, Akzo Nobel, Inc.) and GO $\left(90 \mathrm{~m}^{2} \mathrm{~g}^{-1}\right)$ (Nanjing XFNANO Materials Tech. Co.) instead of PC support, respectively. (3) Fe@C-Glu was obtained in parallel by the same synthesis route as that for Fe-NC SAC, except for no addition of melamine; the samples pyrolyzed 
at 500,600 , and $800^{\circ} \mathrm{C}$ are designated as Fe@C-Glu-500, Fe@C-Glu-600, and Fe@C-Glu, respectively. (4) Fe@C-N was prepared in parallel by the same synthesis route as that for $\mathrm{Fe}-\mathrm{NC} \mathrm{SAC}$, except for no addition of glucose; the samples pyrolyzed at 500 and $800^{\circ} \mathrm{C}$ are designated as Fe@C-N-500 and Fe@C-N, respectively. (5) Fe-NC-Low Glu was prepared in parallel by the same method as that Fe-NC SAC except for using $1.5 \mathrm{mmol}$ glucose instead of $6.7 \mathrm{mmol}$ glucose. (6) $\mathrm{C}-\mathrm{N}-\mathrm{Glu}$ was prepared in parallel by the same synthesis route as that for Fe-NC SAC, except for no addition of iron source. (7) FePc/C was prepared by dispersion $60 \mathrm{mg}$ of PC and $42 \mathrm{mg}$ of FePc (Alfa Aesar Co., Ltd.) in $5 \mathrm{~mL}$ of dimethyl formamide (Alfa Aesar Co., Ltd.), followed by sonication for $30 \mathrm{~min}$ to get a homogenous black suspension. The product was collected after washing and drying. (8) NC was synthesized in parallel by the same method as that Fe-NC SAC, except for no addition of iron source and glucose.

A scale-up synthesis of Fe-NC SAC. The product was prepared in parallel by the same synthesis route as that for Fe-NC SAC, except for increasing the amount of carbon substrate, iron (III) nitrate nonahydrate, glucose, and ultrapure water to $5 \mathrm{~g}$, $24.8 \mathrm{mmol}, 0.56 \mathrm{M}$ and $420 \mathrm{~mL}$, respectively.

Catalyst characterizations. XRD patterns were recorded on Regaku D/Max-2500 (Rigaku Co., Japan) diffractometer equipped with a $\mathrm{Cu} \mathrm{Kal}$ radiation $(\lambda=1.54056$ $\AA$ ). The morphologies were characterized by SEM on S4800 (JEOL, Japan) and TEM on JEM-2100F (JEOL, Japan) equipped with an EDS detector.

HAADF-STEM and EELS mapping observations were carried out on a JEOL ARM200F (JEOL, Japan) STEM operated at $200 \mathrm{kV}$ with cold-filled emission gun and double hexapole Cs correctors (CEOS GmbH, Germany). The attainable spatial resolution defined by the probe-forming objective lens is better than 80 picometers. Nitrogen adsorption-desorption isotherms were collected on a Quadrasorb SI-MP system (Quantachrome, USA) at $77 \mathrm{~K}$. The specific surface area was calculated by Brunauer Emmett Teller method. The pore size distribution and pore volume were calculated using DFT method. XPS spectra were recorded on an ESCALab220i-XL electron spectrometer (VG Scientific, UK) using an $\mathrm{Al} \mathrm{Ka}$ radiation. Raman spectra were obtained on Lab-RAM HR Evolution (Horiba Scientific, Japan) with a laser excitation wavelength of $532 \mathrm{~nm}$. FTIR experiments were performed using Thermo Fisher Nicolet iN10 FTIR microscope (Thermo Nicolet Corp., USA). The metal loading was measured via TGA on a Netzsch DSC214 instrument (NETZSCH, Germany) from 40 to $800{ }^{\circ} \mathrm{C}$ under air flow with a ramp of $10^{\circ} \mathrm{C} \mathrm{min}^{-1}$. XANES and EXAFS of the Fe K-edges were acquired on the XAFS station of the 14W1 beam line of the Shanghai Synchrotron Radiation Facility. The fluorescence mode was used to record the X-ray absorption spectra of Fe K-edges. Data were recorded by using a Si (111) double-crystal monochromator. The back-subtracted EXAFS function was converted into $k$ space and weighted by $k^{3}$ to compensate for the diminishing amplitude due to the decay of the photoelectron wave. The Fourier transforming of the $\mathrm{k}^{3}$-weighted (for $\mathrm{Fe}$ ) EXAFS data was performed in the range of $k=3-12 \AA^{-1}$ using a Hanning function window to get the radial distribution function. The NEXAFS spectra were collected at Beamline 8.0 of Advanced Light Source in Lawrence Berkeley National Lab via a total fluorescence yield mode with a probing depth of $\sim 100 \mathrm{~nm}$. The samples were thoroughly outgassed before measurements.

Electrochemical measurements. ORR Tests. All ORR electrochemical measurements were recorded on a rotating ring-disk electrode rotator (RRDE-3A) (ALS, Japan) by a standard three-electrode cell system connected to an electrochemical workstation (Autolab PGSTAT 302N) (Metrohm, Netherlands) at room temperature. A rotating ring-disk electrode (RRDE) $(4 \mathrm{~mm}$ in diameter) with catalytic material acted as the working electrode; and an $\mathrm{Ag} / \mathrm{AgCl}$ electrode (saturated $\mathrm{KCl}$ solution) and a graphite rod were employed for the reference and counter electrode, respectively. The working electrodes were prepared as follows: all non-precious homogeneous ink was formed by mixing $2 \mathrm{mg}$ of catalysts and $800 \mu \mathrm{L}$ of ethanol (Beijing Chemical Reagent Factory) in a glass vial and sonicated for 30 min. The $30 \mu \mathrm{L}$ of ink and $2 \mu \mathrm{L} 0.5 \mathrm{wt} \%$ of Nafion solution (Alfa Aesar Co., Ltd.) were dropped on polished RRDE to get catalysts loading of $600 \mu \mathrm{g} \mathrm{cm}^{-2}$ and dried $10 \mathrm{~min}$ in the air. For comparison, commercial Johnson-Matthey Pt/C (20 wt\%) was also measured with loading of $25.5 \mu \mathrm{g}_{\mathrm{Pt}} \mathrm{cm}^{-2}$. The accelerated durability tests of Fe-NC SAC and commercial $\mathrm{Pt} / \mathrm{C}$ were performed in the $\mathrm{O}_{2}$-saturated $0.1 \mathrm{M}$ $\mathrm{KOH}$ (Alfa Aesar Co., Ltd.) solution by cycling the catalysts between 0.6 and $1.0 \mathrm{~V}$ at $50 \mathrm{mV} \mathrm{s}^{-1}$, referring to the protocol from USA Department of Energy. Poisoning experiment for $\mathrm{Fe}-\mathrm{NC}$ SAC was first executed in $\mathrm{O}_{2}$-saturated $0.1 \mathrm{M} \mathrm{HClO}_{4}$ (Alfa Aesar Co., Ltd.) with addition of $0.01 \mathrm{M} \mathrm{NaSCN}$ (Alfa Aesar Co., Ltd.). The remarkably depression of catalytic activity can be seen. After that, the working electrode with poisoned $\mathrm{Fe}-\mathrm{NC}$ SAC was rinsed thoroughly and measured again in $0.1 \mathrm{M} \mathrm{KOH}$ under $\mathrm{O}_{2}$ atmosphere. All ORR measurements were collected at a scan rate of $10 \mathrm{mV} \mathrm{s}^{-1}$ at a rotation speed of $1600 \mathrm{rpm}$. Before each ORR test, the electrolyte was purged with oxygen at least for $30 \mathrm{~min}$. All non-Pt catalysts were scanned in the $\mathrm{N}_{2}$-saturated electrolyte. The obtained background voltammograms were subtracted from that measured in the $\mathrm{O}_{2}$-saturated electrolyte before each ORR measurement
The yield of $\mathrm{H}_{2} \mathrm{O}_{2}$ on different catalysts was calculated by the following equation:

$$
\mathrm{H}_{2} \mathrm{O}_{2} \%=200 * \frac{I_{\mathrm{R}} / N}{I_{\mathrm{R}} / N+I_{\mathrm{D}}}
$$

where $I_{\mathrm{R}}$ and $I_{\mathrm{D}}$ are the ring and disk currents, respectively, and $N$ is the ring collection efficiency. The $N$ value was measured to be $42.4 \%$.

$\mathrm{CO}_{2}$ reduction tests. All electrochemical measurements for $\mathrm{CO}_{2}$ reduction were carried out in a gas-tight cell with two-compartments separated with a Nafion membrane (Nafion ${ }^{\circledast} 115$, DuPont, Inc.). A same standard three-electrode cell system as that for ORR was used to collect electrochemical data. In a typical prepared process of the working electrode, $600 \mu \mathrm{L}$ of the mixing ink, which was obtained by dispersing $1 \mathrm{mg}$ catalyst in $30 \mu \mathrm{L} 0.5 \mathrm{wt} \%$ of Nafion solution and $570 \mu \mathrm{L}$ of ethanol solution, was loaded on two sides of a carbon cloth (Alfa Aesar Co., Ltd.) with $1 \times 1 \mathrm{~cm}^{2}$. During the $\mathrm{CO}_{2}$ reduction experiments, all measurements were collected at a scan rate of $10 \mathrm{mV} \mathrm{s}^{-1}$ in $\mathrm{N}_{2}$-saturated $0.5 \mathrm{M} \mathrm{KHCO}_{3}$ (Alfa Aesar Co., Ltd.) or $\mathrm{CO}_{2}$-saturated $0.5 \mathrm{M} \mathrm{KHCO}_{3}$ electrolyte. The gas phase composition was analyzed by gas chromatograph (Agilent 7890B, USA) every $1 \mathrm{~h}$. The separated gas products were measured by a thermal conductivity detector $\left(\right.$ for $\mathrm{H}_{2}$ ) and a flame ionization detector (for $\mathrm{CO}$ ).

\section{Data availability}

The data that support the findings of this study are available from the corresponding author upon reasonable request.

Received: 22 November 2018 Accepted: 1 March 2019

Published online: 20 March 2019

\section{References}

1. Yang, X.-F. et al. Single-atom catalysts: A new frontier in heterogeneous catalysis. Acc. Chem. Res. 46, 1740-1748 (2013).

2. Zhu, C., Fu, S., Shi, Q., Du, D. \& Lin, Y. Single-atom electrocatalysts. Angew. Chem., Int. Ed. 56, 13944-13960 (2017).

3. Herzing, A. A., Kiely, C. J., Carley, A. F., Landon, P. \& Hutchings, G. J. Identification of active gold nanoclusters on iron oxide supports for CO oxidation. Science 321, 1331-1335 (2008).

4. Vajda, S. et al. Subnanometre platinum clusters as highly active and selective catalysts for the oxidative dehydrogenation of propane. Nat. Mater. 8, 213-216 (2009).

5. Jones, J. et al. Thermally stable single-atom platinum-on-ceria catalysts via atom trapping. Science 353, 150-154 (2016).

6. $\mathrm{Li}, \mathrm{H}$. et al. Synergetic interaction between neighbouring platinum monomers in $\mathrm{CO}_{2}$ hydrogenation. Nat. Nanotechnol. 13, 411-417 (2018).

7. Yang, F., Deng, D., Pan, X., Fu, Q. \& Bao, X. Understanding nano effects in catalysis. Natl. Sci. Rev. 2, 183-201 (2015).

8. Qiao, B. et al. Single-atom catalysis of $\mathrm{CO}$ oxidation using $\mathrm{Pt}_{1} / \mathrm{FeO}_{\mathrm{x}}$. Nat. Chem. 3, 634-641 (2011).

9. Liu, P. et al. Photochemical route for synthesizing atomically dispersed palladium catalysts. Science 352, 797-800 (2016).

10. Shan, J., Li, M., Allard, L. F., Lee, S. \& Flytzani-Stephanopoulos, M. Mild oxidation of methane to methanol or acetic acid on supported isolated rhodium catalysts. Nature 551, 605-608 (2017).

11. Peterson, E. J. et al. Low-temperature carbon monoxide oxidation catalysed by regenerable atomically dispersed palladium on alumina. Nat. Commun. 5, 4885 (2014).

12. Ding, $\mathrm{K}$. et al. Identification of active sites in $\mathrm{CO}$ oxidation and water-gas shift over supported Pt catalysts. Science 350, 189-192 (2015).

13. Lin, J. et al. Remarkable performance of $\mathrm{Ir}_{1} / \mathrm{FeO}_{\mathrm{x}}$ single-atom catalyst in water gas shift reaction. J. Am. Chem. Soc. 135, 15314-15317 (2013).

14. Guo, X. et al. Direct, nonoxidative conversion of methane to ethylene, aromatics, and hydrogen. Science 344, 616-619 (2014).

15. $\mathrm{Li}, \mathrm{X}$. et al. Single-atom $\mathrm{Pt}$ as co-catalyst for enhanced photocatalytic $\mathrm{H}_{2}$ evolution. Adv. Mater. 28, 2427-2431 (2016).

16. Gao, G., Jiao, Y., Waclawik, E. R. \& Du, A. Single atom (Pd/Pt) supported on graphitic carbon nitride as an efficient photocatalyst for visible-light reduction of carbon dioxide. J. Am. Chem. Soc. 138, 6292-6297 (2016).

17. Cheng, $\mathrm{N}$. et al. Platinum single-atom and cluster catalysis of the hydrogen evolution reaction. Nat. Commun. 7, 13638 (2016).

18. Bayatsarmadi, B., Zheng, Y., Vasileff, A. \& Qiao, S.-Z. Recent advances in atomic metal doping of carbon-based nanomaterials for energy conversion. Small 13, 1700191 (2017).

19. Wei, H. et al. Iced photochemical reduction to synthesize atomically dispersed metals by suppressing nanocrystal growth. Nat. Commun. 8, 1490 (2017). 
20. Chung, H. T. et al. Direct atomic-level insight into the active sites of a highperformance PGM-free ORR catalyst. Science 357, 479-484 (2017).

21. Yin, P. et al. Single cobalt atoms with precise $\mathrm{N}$-coordination as superior oxygen reduction reaction catalysts. Angew. Chem., Int. Ed. 55, 10800-10805 (2016).

22. Han, A. et al. A polymer encapsulation strategy to synthesize porous nitrogendoped carbon-nanosphere-supported metal isolated-single-atomic-site catalysts. Adv. Mater. 30, 1706508 (2018).

23. Liu, J. et al. High performance platinum single atom electrocatalyst for oxygen reduction reaction. Nat. Commun. 8, 15938 (2017).

24. Wang, J. et al. In situ formation of molecular Ni-Fe active sites on heteroatomdoped graphene as a heterogeneous electrocatalyst toward oxygen evolution. Sci. Adv. 4, eaap7970 (2018).

25. Ju, W. et al. Understanding activity and selectivity of metal-nitrogen-doped carbon catalysts for electrochemical reduction of $\mathrm{CO}_{2}$. Nat. Commun. 8, 944 (2017).

26. Shi, M.-M. et al. Au sub-nanoclusters on $\mathrm{TiO}_{2}$ toward highly efficient and selective electrocatalyst for $\mathrm{N}_{2}$ conversion to $\mathrm{NH}_{3}$ at ambient conditions. Adv. Mater. 29, 1606550 (2017).

27. Shi, Y. et al. Single-atom catalysis in mesoporous photovoltaics: The principle of utility maximization. Adv. Mater. 26, 8147-8153 (2014).

28. Judai, K., Abbet, S., Wörz, A. S., Heiz, U. \& Henry, C. R. Low-temperature cluster catalysis. J. Am. Chem. Soc. 126, 2732-2737 (2004).

29. Lee, S., Fan, C., Wu, T. \& Anderson, S. L. CO oxidation on $\mathrm{Au}_{\mathrm{n}} / \mathrm{TiO}_{2}$ catalysts produced by size-selected cluster deposition. J. Am. Chem. Soc. 126, $5682-5683$ (2004).

30. Lei, Y. et al. Increased silver activity for direct propylene epoxidation via subnanometer size effects. Science 328, 224-228 (2010).

31. Liu, L. et al. Generation of subnanometric platinum with high stability during transformation of a 2D zeolite into 3D. Nat. Mater. 16, 132-138 (2017).

32. Choi, C. H. et al. Tuning selectivity of electrochemical reactions by atomically dispersed platinum catalyst. Nat. Commun. 7, 10922 (2016).

33. Fei, H. et al. Atomic cobalt on nitrogen-doped graphene for hydrogen generation. Nat. Commun. 6, 8668 (2015).

34. Malko, D., Kucernak, A. \& Lopes, T. In situ electrochemical quantification of active sites in Fe-N/C non-precious metal catalysts. Nat. Commun. 7, 13285 (2016).

35. Li, Y. et al. An oxygen reduction electrocatalyst based on carbon nanotube-graphene complexes. Nat. Nanotechnol. 7, 394-400 (2012).

36. Genovese, C. et al. Operando spectroscopy study of the carbon dioxide electro-reduction by iron species on nitrogen-doped carbon. Nat. Commun. 9, 935 (2018).

37. Xu, H., Cheng, D., Cao, D. \& Zeng, X. C. A universal principle for a rational design of single-atom electrocatalysts. Nat. Catal. 1, 339-348 (2018).

38. Deng, D. et al. A single iron site confined in a graphene matrix for the catalytic oxidation of benzene at room temperature. Sci. Adv. 1, e1500462 (2015).

39. Liu, W. et al. Single-atom dispersed Co-N-C catalyst: Structure identification and performance for hydrogenative coupling of nitroarenes. Chem. Sci. 7 , 5758-5764 (2016).

40. Chen, Z. et al. Stabilization of single metal atoms on graphitic carbon nitride. Adv. Funct. Mater. 27, 1605785 (2017)

41. Fei, H. et al. General synthesis and definitive structural identification of $\mathrm{MN}_{4} \mathrm{C}_{4}$ single-atom catalysts with tunable electrocatalytic activities. Nat. Catal. 1, 63-72 (2018)

42. Campbell, C. T., Parker, S. C. \& Starr, D. E. The effect of size-dependent nanoparticle energetics on catalyst sintering. Science 298, 811-814 (2002)

43. Simonsen, S. B. et al. Direct observations of oxygen-induced platinum nanoparticle ripening studied by in situ TEM. J. Am. Chem. Soc. 132, 7968-7975 (2010).

44. Hansen, T. W., DeLaRiva, A. T., Challa, S. R. \& Datye, A. K. Sintering of catalytic nanoparticles: Particle migration or ostwald ripening? Acc. Chem. Res. 46, 1720-1730 (2013).

45. Risse, T., Shaikhutdinov, S., Nilius, N., Sterrer, M. \& Freund, H.-J. Gold supported on thin oxide films: From single atoms to nanoparticles. Acc. Chem. Res. 41, 949-956 (2008).

46. Geetha, K., Raghavan, M. S. S., Kulshreshtha, S. K., Sasikala, R. \& Rao, C. P. Transition-metal saccharide chemistry: Synthesis, spectroscopy, electrochemistry and magnetic susceptibility studies of iron(III) complexes of mono- and disaccharides. Carbohydr. Res. 271, 163-175 (1995).

47. Nagy, L. et al. Iron(III) complexes of sugar-type ligands. Inorg. Chim. Acta 124, 55-59 (1986).

48. Nagy, L., Gajda, T., Kürti, J., Schrantz, K. \& Burger, K. Spectroscopic studies of iron(III) complexes of D-saccharose and D-glucose in the solid state and in solution. J. Radioanal. Nucl. Chem. 209, 225-234 (1996).

49. Jiang, R. et al. Edge-site engineering of atomically dispersed $\mathrm{Fe}-\mathrm{N}_{4}$ by selective $\mathrm{C}-\mathrm{N}$ bond cleavage for enhanced oxygen reduction reaction activities. J. Am. Chem. Soc. 140, 11594-11598 (2018).
50. Cao, R. et al. Promotion of oxygen reduction by a bio-inspired tethered iron phthalocyanine carbon nanotube-based catalyst. Nat. Commun. 4, 2076 (2013).

51. Cheon, J. Y. et al. Graphitic nanoshell/mesoporous carbon nanohybrids as highly efficient and stable bifunctional oxygen electrocatalysts for rechargeable aqueous Na-air batteries. Adv. Energy Mater. 6, 1501794 (2016).

52. Zhou, J. et al. Fe-N bonding in a carbon nanotube-graphene complex for oxygen reduction: an XAS study. Phys. Chem. Chem. Phys. 16, 15787-15791 (2014).

53. Cook, P. L., Liu, X., Yang, W. \& Himpsel, F. J. X-ray absorption spectroscopy of biomimetic dye molecules for solar cells. J. Chem. Phys. 131, 194701 (2009).

54. Yang, J. et al. In situ thermal atomization to convert supported nickel nanoparticles into surface-bound nickel single-atom catalysts. Angew. Chem., Int. Ed. 57, 14095-14100 (2018).

55. Wang, X. et al. Uncoordinated amine groups of metal-organic frameworks to anchor single Ru sites as chemoselective catalysts toward the hydrogenation of quinoline. J. Am. Chem. Soc. 139, 9419-9422 (2017).

56. Li, X. et al. Simultaneous nitrogen doping and reduction of graphene oxide. J. Am. Chem. Soc. 131, 15939-15944 (2009).

57. Zou, X. et al. Cobalt-embedded nitrogen-rich carbon nanotubes efficiently catalyze hydrogen evolution reaction at all $\mathrm{pH}$ values. Angew. Chem., Int. Ed. 53, 4372-4376 (2014).

58. Wang, H., Maiyalagan, T. \& Wang, X. Review on recent progress in nitrogendoped graphene: Synthesis, characterization, and its potential applications. ACS Catal. 2, 781-794 (2012).

59. Yang, J., Liu, D.-J., Kariuki, N. N. \& Chen, L. X. Aligned carbon nanotubes with built-in $\mathrm{FeN}_{4}$ active sites for electrocatalytic reduction of oxygen. Chem. Commun. 21, 329-331 (2008).

60. Wu, G. et al. Synthesis-structure-performance correlation for polyaniline-Me-C non-precious metal cathode catalysts for oxygen reduction in fuel cells. J. Mater. Chem. 21, 11392-11405 (2011).

61. Wu, Z. Y. et al. Characterization of iron oxides by $\mathrm{x}$-ray absorption at the oxygen K edgeusing a full multiple-scattering approach. Phys. Rev. B 55, 2570-2577 (1997)

62. Yan, S. C., Li, Z. S. \& Zou, Z. G. Photodegradation performance of $\mathrm{g}^{-} \mathrm{C}_{3} \mathrm{~N}_{4}$ fabricated by directly heating melamine. Langmuir 25, 10397-10401 (2009).

63. Wei, S. et al. Direct observation of noble metal nanoparticles transforming to thermally stable single atoms. Nat. Nanotechnol. 13, 856-861 (2018).

64. Wu, G., More, K. L., Johnston, C. M. \& Zelenay, P. High-performance electrocatalysts for oxygen reduction derived from polyaniline, iron, and cobalt. Science 332, 443-447 (2011).

65. Shui, J., Wang, M., Du, F. \& Dai, L. N-doped carbon nanomaterials are durable catalysts for oxygen reduction reaction in acidic fuel cells. Sci. Adv. 1, e1400129 (2015).

66. Wang, Q. et al. Phenylenediamine-based $\mathrm{FeN}_{\mathrm{x}} / \mathrm{C}$ catalyst with high activity for oxygen reduction in acid medium and its active-site probing. J. Am. Chem. Soc. 136, 10882-10885 (2014)

67. Jiang, W.-J. et al. Understanding the high activity of $\mathrm{Fe}-\mathrm{N}-\mathrm{C}$ electrocatalysts in oxygen reduction: $\mathrm{Fe} / \mathrm{Fe}_{3} \mathrm{C}$ nanoparticles boost the activity of $\mathrm{Fe}-\mathrm{N}_{\mathrm{x}}$. J. Am. Chem. Soc. 138, 3570-3578 (2016).

68. Chen, $\mathrm{Y}$. et al. Isolated single iron atoms anchored on $\mathrm{N}$-doped porous carbon as an efficient electrocatalyst for the oxygen reduction reaction. Angew. Chem., Int. Ed. 56, 6937-6941 (2017).

69. Li, Q. et al. Metal-organic framework-derived bamboo-like nitrogen-doped graphene tubes as an active matrix for hybrid oxygen-reduction electrocatalysts. Small 11, 1443-1452 (2015).

\section{Acknowledgements}

This work was supported by the National Key Project on Basic Research (2015CB932302), National Natural Science Foundation of China (21773263 and 91645123) and the Strategic Priority Research Program of the Chinese Academy of Sciences (XDB12020100). This research used resources of the Advanced Light Source, which is a DOE Office of Science User Facility under contract no. DE-AC02-05CH11231. Any opinions, findings, conclusions or recommendations expressed in this work are those of the author(s) and do not necessarily reflect the views of the National Natural Science Foundation of China, Strategic Priority Research Program of the Chinese Academy of Sciences.

\section{Author contributions}

L.Z., Y. Z. and J.S.H. conceived the project. L.Z., L.B.H. and C.H. carried out the synthesis most of the structural characterizations and electrochemical tests. X.Z.L., Q.H.Z. and L.G. performed the HAADF-STEM characterizations and EELS experiments. L.J.Z. analyzed the EXAFS and XANES data. Z.Y.W. carried out DFT calculations. J.W. and W.Y. performed NEXFAS experiments. L.J.W. discussed the results and commented on the paper. L.Z., Y. Z. and J.S.H. cowrote the paper. J.S.H. supervised the project. 


\section{Additional information}

Supplementary Information accompanies this paper at https://doi.org/10.1038/s41467019-09290-y.

Competing interests: The authors declare no competing interests.

Reprints and permission information is available online at http://npg.nature.com/ reprintsandpermissions/

Journal peer review information: Nature Communications thanks the anonymous reviewers for their contribution to the peer review of this work. Peer reviewer reports are available.

Publisher's note: Springer Nature remains neutral with regard to jurisdictional claims in published maps and institutional affiliations. (c) (i) Open Access This article is licensed under a Creative Commons Attribution 4.0 International License, which permits use, sharing, adaptation, distribution and reproduction in any medium or format, as long as you give appropriate credit to the original author(s) and the source, provide a link to the Creative Commons license, and indicate if changes were made. The images or other third party material in this article are included in the article's Creative Commons license, unless indicated otherwise in a credit line to the material. If material is not included in the article's Creative Commons license and your intended use is not permitted by statutory regulation or exceeds the permitted use, you will need to obtain permission directly from the copyright holder. To view a copy of this license, visit http://creativecommons.org/ licenses/by/4.0/.

(C) The Author(s) 2019 